\title{
Optical mode conversion in coupled Fabry-Pérot resonators
}

\author{
Mark Stone ${ }^{1}$, Aziza Suleymanzade ${ }^{1}$, Lavanya Taneja ${ }^{1}$, David Schuster ${ }^{1}$, and Jonathan Simon ${ }^{1}$ \\ ${ }^{1}$ Department of Physics and James Franck Institute, University of Chicago, Chicago, IL
}

(Dated: May 26, 2020)

\begin{abstract}
Coherent control of the spatial properties of light is central to a wide variety of applications from high bandwidth quantum [1 3] and classical [4 6] communication to high power fiber lasers [7. Lowloss conversion amongst a complete and orthogonal set of modes is particularly important for robust mode-multiplexed communication [8, 9. Here, we introduce tunable impedance mismatch [10] between coupled Fabry-Pérot resonators as a powerful tool for manipulation of the spatial and temporal properties of optical fields. In the single-mode regime, frequency dependent impedance matching enables tunable finesse optical resonators, with potential applications in quantum science and sensing. Introducing the spatial dependence of the impedance mismatch as an additional ingredient enables coherent spatial mode conversion of optical photons at near-unity efficiency. We implement these ideas, experimentally demonstrating a NIR resonator whose finesse is tunable over a decade, and an optical mode converter with efficiency $>75 \%$ for the first six Hermite-Gauss modes. We anticipate that this new perspective on coupled multimode resonators will have exciting applications in micro- 11, 12] and nano- [13 16] photonics and computer-aided inverse design [17. In particular, combination with in-cavity electro-optics [18, 19] will open new horizons for real-time control of the spatio-spectral properties of lasers, resonators, and optical filters.
\end{abstract}

\section{A. INTRODUCTION}

High fidelity mode conversion and sorting are crucial tasks for quantum communication [1-3, 8, as well as high-bandwidth mode-division multiplexed classical communication [4]. At the transmitting end of a communication network, mode conversion enables the encoding of information into the transverse spatial degrees of freedom of an optical field or fiber, thereby substantially increasing the bit-rate. At the receiving end, modesorting enables decoding of the previously encoded spatial information. While both mode conversion and sorting are fundamentally linear in the electromagnetic field, they are technically challenging because the necessary linear transformations are not generically quadratic in the transverse spatial coordinates and as such cannot be directly implemented with standard optics like mirrors, lenses, and beam splitters.

At moderate efficiency, "mode shaping" can be achieved with a single phase plate [20 22 or digital micromirror device [23, 24] that redirects a fraction of an incident optical field into a diffracted target mode; an additional spatial filter may be used to remove power in undesired modes 25. Near-unity efficiency requires implementing a unitary transformation of all of the incident mode to the target mode. In the special case of Hermite-Gauss $\leftrightarrow$ Laguerre-Gauss inter-conversion, this unitary transformation can be realized via a pair of astigmatic lenses [26], since $\mathrm{HG}$ and $\mathrm{LG}$ modes are related to one another by only the relative phase of horizontal and vertical mode excitations. More general approaches to high fidelity mode-converting unitaries include numerically optimized nanostructured couplers between waveguides [27, 28]; adiabatically varying coupling between macroscopic optical fibers [29, 30]; conformal beam transformations implemented in two or more holographic phase gratings [9, 31,34]; meshes of Mach-
Zehnder interferometers [35, 36]; and long period fiber gratings [37, 38].

Implementing an arbitrary mode converter is formally equivalent to changing one quantum mechanical wavefunction into another using only spatially local potentials which cannot themselves redistribute probability in space, but can impose phase gradients that result in such redistribution under the influence of a kinetic energy term. While lenses and mirrors can impart spatially varying phase profiles onto an incident optical field, it is the subsequent diffraction that must redistribute intensity; reshaping the mode via attenuation would irreversibly reduce the conversion efficiency. To our knowledge, all prior work fits within one of three paradigms: adiabatically varying the system Hamiltonian such that an input mode/initial eigenstate is smoothly converted into the desired output mode/final eigenstate (equivalent to coupled fibers); bang-bang unitaries that, in discrete steps separated by free-evolution/diffraction, convert between input and output modes (equivalent to cascaded diffraction gratings or long-period fiber gratings); or something in-between that implements a "shortcut to adiabaticity" 39.

Here we present a new approach that breaks this paradigm and instead relies upon impedance mismatches between optical cavities to achieve near-unity efficiency mode conversion without nanophotonics or nonquadratic optics. Using only lenses and mirrors, we demonstrate conversion of an $\mathrm{HG}_{00}$ mode into an arbitrary target $\mathrm{HG}_{m 0}$ mode by simply varying the length of a Fabry-Pérot resonator over a few nanometers. The large propagation distances required for prior approaches are realized in our work by repeated round trips through the complex structure of the coupled cavities.

In Section B we introduce the simpler problem of coupled, impedance-mismatched Fabry-Pérot cavities in the single-mode limit. Here the result is a cavity of tunable 
finesse $\mathcal{F}$. We then experimentally demonstrate such finesse tunability over a decade and characterize its properties in comparison with an S-matrix analysis. In Section C we consider the full problem of coupled, misaligned multimode Fabry-Pérot cavities, where the resulting behaviour corresponds to an optical mode converter. Implementing these ideas, we demonstrate optical conversion efficiency $>75 \%$ for the first 6 Hermite-Gauss modes, limited by mirror loss and accidental mode-degeneracies. In Section D we explore applications and outlook for these new tools.

\section{B. TUNABLE FINESSE CAVITY}

We begin by analyzing two single-mode Fabry-Pérot cavities coupled through a shared mirror, as shown in Fig. 17. We will find that this arrangement acts as a tunable finesse cavity - it traps light for a short duration (low finesse) or a long duration (high finesse). The two cavities have identical waists and share a mutual axis to avoid inter-mode coupling.

The total optical transmission of this arrangement can be calculated in the S-matrix formalism (see Appendix A in terms of the lengths of the two cavities, the wavenumber $k$ of incident light, and the power reflection and transmission coefficients of the mirrors $M_{1}, M_{2}$, and $M_{3}$. A more intuitive understanding arises by observing that any single-mode scattering element is fully described by its (frequency $\delta \equiv 2 k L$ dependent) reflection and transmission coefficients. It is thus valid to combine mirrors $M_{2}$ and $M_{3}$ with the propagation distance $L$ between them, into a single composite "effective mirror" $M_{23}$ with reflection and transmission coefficients $r_{23}(\delta)$, $t_{23}(\delta)$ (Fig. 17a, inset).

In this picture, what remains is the simple two-mirror "primary" cavity defined by the separation between $M_{1}$ and $M_{23}$. The total transmission of the primary cavity is thus precisely that of a simple two-mirror Fabry-Pérot with a frequency-dependent reflection coefficient for one end-mirror. The finesse of the primary cavity can be computed according to 41 as $\mathcal{F} \approx \frac{2 \pi}{T_{1}+T_{23}+X_{1}+X_{23}}$ so long as the properties of $M_{23}$ remain $\sim$ constant across said resonance. Here $T_{1}$ and $T_{23}$ are power transmission coefficients and $X_{1}, X_{23}$ are power loss coefficients. As $T_{23}$ is tuned, the finesse $\mathcal{F}$ varies. Since $T_{23}$ is simply the transmission of the Fabry-Pérot consisting of $M_{2}$ and $M_{3}$, it can range from unity to near zero as the length $L$ tunes the cavity from resonance to antiresonance, thereby varying $\mathcal{F}$ from small to large values.

Harnessing these principles, we construct a tunable finesse cavity using mirrors with reflectances $R_{1}=$ 0.9997(1) and $R_{2}=R_{3}=0.990(2)$ for $780 \mathrm{~nm}$ light. The finesse is tuned by varying $M_{23}$ 's length with a piezoelectric actuator, and is then measured either spectroscopically or by cavity ringdown 40] (Fig. 11). The computed transmission of $M_{23}, T_{23}$ is shown in the black curve of Fig. 17, varying from unity on resonance to
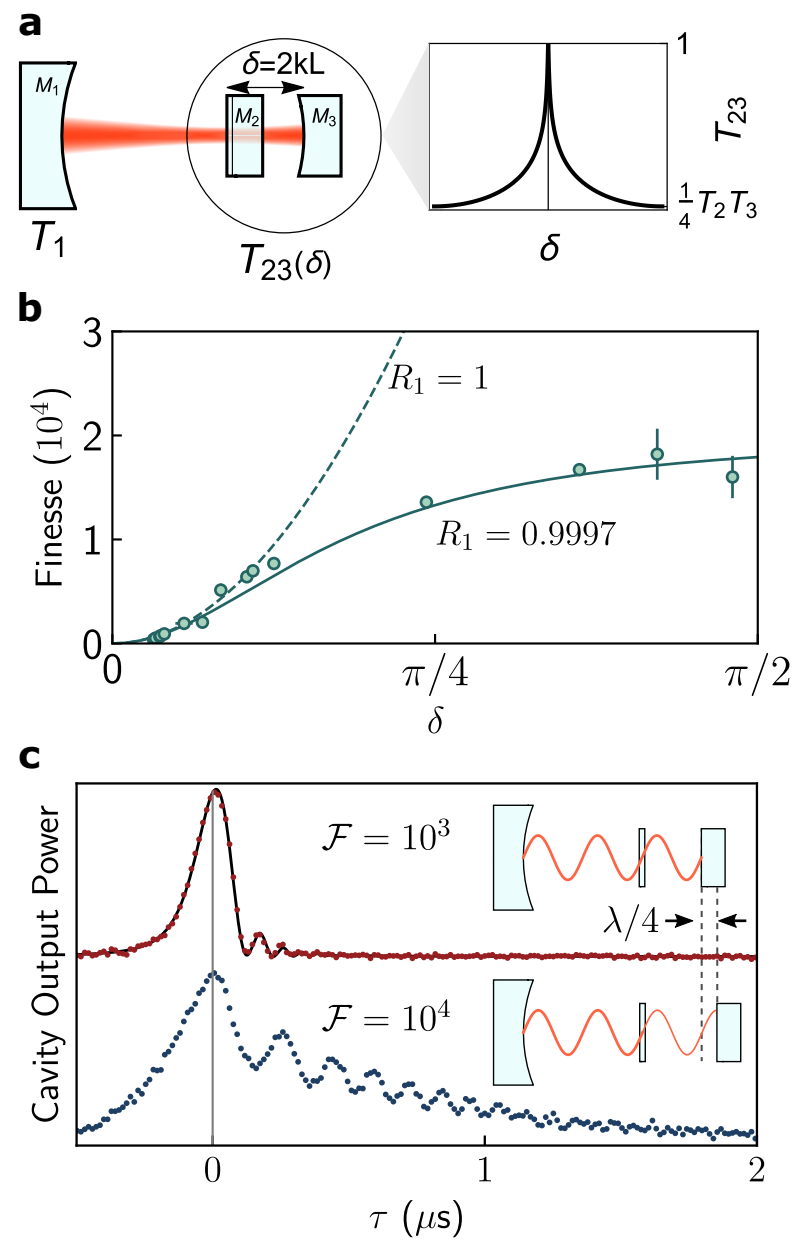

FIG. 1. Tunable finesse optical cavity. a, Schematic of two coupled single-mode cavities. Mirrors $M_{2}$ and $M_{3}$ act as a single "effective mirror" $M_{23}$ with frequency-dependent transmission $T_{23}(\delta)$, where $\delta \equiv 2 k L$ is the round-trip propagation phase in $M_{23}$. Sub- $\lambda$ variations in the $M_{2}-M_{3}$ separation change their joint transmission, and thereby the finesse $\mathcal{F}$ of the composite $M_{1}-M_{23}$ cavity. $T_{23}$ varies from unity at resonance to $\frac{1}{4} T_{2} T_{3}$ between resonances $\left(T_{i}\right.$ is the power transmission of mirror $i$ ). $\mathbf{b}$, Measured $\mathcal{F}$ of the $M_{1}-M_{23}$ cavity as a function of the round-trip optical phase $\delta$ in $M_{23}$, obtained from cavity ringdowns (for high $\mathcal{F}$ ) and transmission spectra (for lowest $\mathcal{F}$ ). The solid line is theory from measured mirror reflectances, limited by the reflectance $\left(R_{1}=0.9997(1)\right)$ of $M_{1}\left(R_{1}=1\right.$ shown dashed). Error bars represent 1 s.d. of finesse. c, Typical low- and high- $\mathcal{F}$ ringdown measurements with representative exp-erfc fit 40. (black line). Top inset: the cavities are mutually resonant (support an integer number of half wavelengths $\lambda / 2$ ) for low $\mathcal{F}$. Bottom inset: displacing $M_{3}$ by a $\lambda / 4$ shifts $M_{23}$ off resonance, reducing its transmission and achieving high $\mathcal{F}$.

$\frac{1}{4} T_{2} T_{3} \approx 2.5 \times 10^{-5}$ at maximum detuning. The measured finesse is shown in Fig. 1 $\mathrm{b}$, in close agreement with a parameter-free theory (solid curve).

The finesse saturates at $1.7(2) \times 10^{4}$, limited by the reflectance of $M_{1}$, and is compared to theory for a perfect $M_{1}\left(R_{1}=1\right)$ in the dotted curve of Fig. 1 p. From there, 
the next bound on finesse is set by the minimum transmission of the variable reflector, $\mathcal{F}_{\max }=\frac{8 \pi}{T_{2} T_{3}} \approx 2.5 \times 10^{5}$ for $R_{2}=R_{3}=0.99$. In practice, we anticipate an ultimate finesse limit set by scattering and absorption losses of the mirror coatings [42, akin to a conventional FabryPérot cavity (see Appendix A). Since the $M_{2}$ substrate lies within the resonator, one might anticipate that absorption in the glass would strongly limit $\mathcal{F}$. However, in high-finesse configurations, very little power resides within $M_{23}$, so losses from the $M_{2}$ substrate and $M_{3}$ coating are strongly suppressed. A single-pass substrate loss of $1 \%(0.1 \%)$ only limits $\mathcal{F} \leq 1 \times 10^{5}\left(2 \times 10^{5}\right)$, which improves further with higher $R_{2}, R_{3}$. Furthermore, fusedsilica glasses can exhibit losses below $1 \mathrm{ppm} / \mathrm{cm}$ [43], entirely obviating this limitation.

When the detuning between the cavities is smaller than the linewidth of the secondary cavity, the above picture breaks down, because (a) the $M_{23}$ transmission $T_{23}$ becomes strongly frequency dependent, or equivalently (b) there is an avoided crossing between the two coupled cavity modes. In practice, this means that the round-trip loss of the primary cavity cannot exceed $T_{1}+T_{3}$.

Imperfect mode matching leads to leakage of light out of the $M_{1}-M_{23}$ cavity through higher-order modes of $M_{23}$, potentially limiting the maximum achievable finesse. As with leakage through the lowest mode of $M_{23}$, this loss is suppressed as the modes are detuned from the primary cavity resonance. By making the $M_{23}$ cavity highly degenerate, it is possible for the fundamental mode of the primary cavity to be spectrally isolated from all modes of the $M_{23}$ cavity, thus avoiding accidental neardegeneracies. We choose $L$ to realize a half-confocal cavity with $\omega_{n l m}=\omega_{f s r}\left[n+\frac{1}{4}(l+m)\right]$, ensuring that the mode of the primary cavity is detuned by at least $1 / 8$ of a free spectral range (FSR) from all modes of $M_{23}$. This detuning results in a transmission suppression of $\frac{2-\sqrt{2}}{4} \approx 15 \%$ relative to that at a detuning of $1 / 2$ the FSR: as long as the mode matching is better than $85 \%$, the maximum finesse should not be significantly affected.

\section{MODE CONVERTER}

Coupled optical cavities, as shown in Fig. 2al, enable near-unity efficiency mode conversion by a multimode generalization of single-mode impedance matching: at resonance, a two-mirror, single-mode cavity with equal in- and out- coupling $T_{1}=T_{2}$ transmits all light. The two coupled resonators explored in the prior section can be understood as one single-mode cavity with input coupling $T_{1}$ and (frequency-dependent) output coupling $T_{23}(\omega)$ of the composite mirror $M_{23}$. Unity transmission again occurs when in- and out- couplings are equal, $T_{1}=T_{23}(\omega)$. Because $T_{23}$ exhibits a resonance peak whose center frequency can be tuned by adjusting the length $L$ between $M_{2}$ and $M_{3}$, it is always possible to satisfy the impedance matching condition for a given drive frequency $\omega=\omega_{d}$.

In the absence of transverse mode coupling, an in-

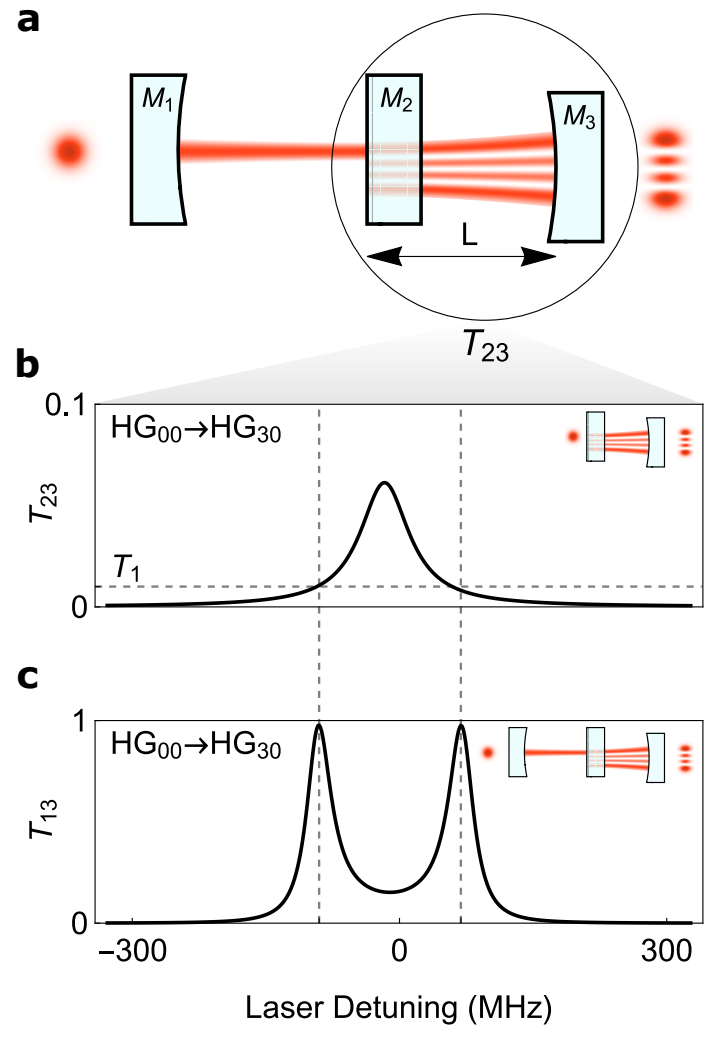

FIG. 2. Principle of Optical Mode Conversion. a, Two coupled Fabry-Pérot resonators can act as an optical mode converter when a small transverse offset is introduced between their axes to couple their otherwise-orthogonal transverse modes. Mirrors $M_{2}$ and $M_{3}$ act as a single "effective mirror" $M_{23}$ with frequency- and mode- dependent transmission $T_{23}^{i \leftrightarrow j}$, for input/output modes $\mathrm{HG}_{i / j, 0}$. Near-unity efficiency $i \leftrightarrow j$ mode conversion through the full system $M_{1}+M_{23}$ is achieved when the input- and output- couplings to the composite cavity $M_{1} / M_{23}$ are equal, $T_{1}=T_{23}^{i \leftrightarrow j}$ (the "impedance matching" condition), and no light leaks out through other modes. b, Simulated transmission of the effective mirror $M_{23}$ (in the absence of $M_{1}$ ), with a translated $\mathrm{HG}_{0,0}$ input generating an $\mathrm{HG}_{3,0}$ output. The transmission $T_{23}^{0 \leftrightarrow 3}$ is limited by the (translated) $0 / 3$ mode overlap of $\approx 6 \%$, and the dashed horizontal line denotes $T_{1}$. The frequency dependence of the transmission guarantees that there are two frequencies where $T_{1}=T_{23}^{0 \leftrightarrow 3}$ (dashed vertical lines), resulting in perfect mode conversion at these frequencies once mirror $M_{1}$ is introduced, as shown in $\mathbf{c}$.

put $\mathrm{HG}_{m} \equiv \mathrm{HG}_{m 0}$ mode produces an output $\mathrm{HG}_{m}$ mode, and the single-mode analysis applies. Introducing a transverse offset between the coupled cavities breaks orthogonality between their higher-order modes and generates inter-mode couplings (Fig.2 2 ). In this case, the $\mathrm{HG}_{0}$ mode of the primary cavity appears displaced on $M_{23}$, and thus has non-zero overlap with all modes of $M_{23}$. As such, $M_{23}$ now exhibits frequency- and mode- dependent transmission $T_{23}^{i \leftrightarrow j}(\omega)=\left|\alpha_{i j}\right|^{2} T_{23}^{j}(\omega)$, with input and output modes $i$ and $j$ having an overlap integral $\alpha_{i j}$. The transverse modes of $M_{23}$ each have their own trans- 


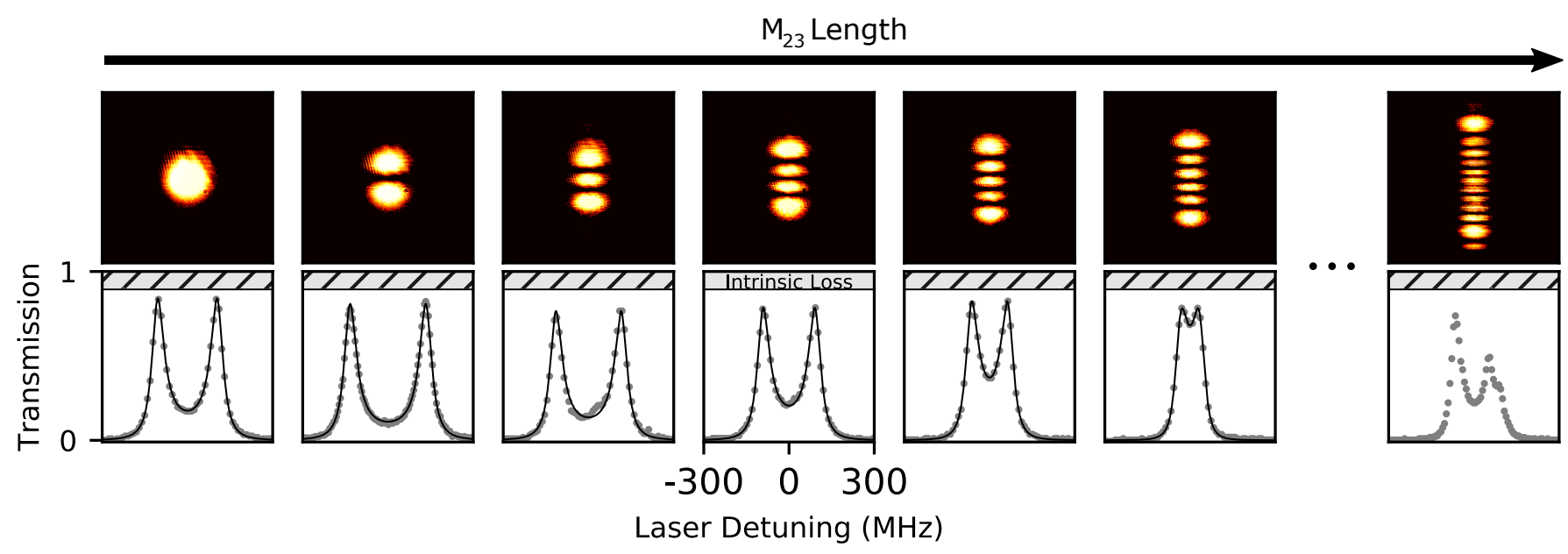

FIG. 3. Demonstration of High-Efficiency Mode Conversion. An input $\mathrm{HG}_{00}$ mode may be coherently converted into any higher order $\mathrm{HG}_{m 0}$ mode by using two coupled, transversely offset Fabry-Pérot cavities. The output spatial profile (top) and end-to-end conversion efficiency (bottom) are plotted for output modes $\mathrm{HG}_{00} \ldots \mathrm{HG}_{50}$ and $\mathrm{HG}_{10,0}$. As the length of output cavity $M_{23}$ is tuned with a piezoelectric actuator, its higher-order modes are individually brought near resonance with the drive laser. Each resonant mode of the $M_{23}$ cavity satisfies the impedance matching condition at two laser frequencies (Fig. 2), giving two peaks with near-unity efficiency mode conversion. In each panel the laser frequency is swept by $\pm 300 \mathrm{MHz}$ around the mutual resonance, demonstrating a mode-conversion bandwidth of $\sim 50 \mathrm{MHz}$. Mirror absorption and scattering limits the maximum conversion efficiency (hashed region). Optical power may be diverted into other accidentally degenerate modes, reducing conversion into the target mode and producing additional peaks and asymmetry in the transmission spectrum. Model fits (solid lines) are consistent with a transverse offset between cavities of 1.30(1) waists.

mission function $T_{23}^{j}(\omega)$, all with identical linewidths, but different resonant frequencies due to the round-trip Gouy phase of $M_{23}$ [41]. The simulated $\mathrm{HG}_{0} \rightarrow \mathrm{HG}_{3}$ transmission peak is shown in Fig. $2 \mathrm{p}$.

We expect unity transmission to occur when $T_{1}=$ $T_{23}^{i \leftrightarrow j}\left(\omega_{d}\right)$, where in-coupling occurs through the $\mathrm{HG}_{i}$ mode at $M_{1}$, and out-coupling occurs through the $\mathrm{HG}_{j}$ mode of $M_{23}$. The multimode S-Matrix calculation shown in Fig. 22 supports this intuition, showing nearly $100 \%$ conversion efficiency. As the length of $M_{23}, L$, is tuned, its higher order modes individually approach resonance with the drive laser and primary cavity, satisfying the impedance matching condition at two drive-laser frequencies and thus permitting conversion of any input mode $i$ into any output so long as $\left|\alpha_{i j}\right|^{2}>T_{1}$. Indeed, for the theory in Fig. 2, the mode overlap between $\mathrm{HG}_{0}$ and $\mathrm{HG}_{3}$ is only $\sim 6 \%$, but near-unity conversion still occurs.

To demonstrate these principles, we construct a mode converter using mirrors with reflectances $R_{1}=R_{3}=$ $0.965(5), R_{2}=0.972(1)$ at $780 \mathrm{~nm}$, whose performance is shown in Fig. 3. In each panel, the laser frequency is scanned to satisfy the resonance condition. Between panels, the length $L$ of $M_{23}$ is varied with a piezoelectric actuator to bring the target mode to resonance with the $\mathrm{HG}_{0}$ mode of the primary cavity. The transmission is monitored on a large-area photodiode to determine conversion efficiency, and on a CCD camera to ascertain mode shape. With the cavities transversely offset by $\sim 1$ waist, $\mathrm{HG}_{0}$ through $\mathrm{HG}_{5}$ were generated with total conversion/transmission in excess of $75 \%$. To access higher order modes, the offset was increased to $\sim 2.5$ waists and the piezo scanned as before, yielding conversion of modes up to $\mathrm{HG}_{12}$; the $\mathrm{HG}_{10}$ mode is shown with $75 \%$ total transmission.

\section{OUTLOOK}

We have presented a new paradigm for understanding coupled optical resonators, where one resonator acts as a frequency- and/or mode- dependent mirror for the other resonator. Harnessing this new perspective, we have demonstrated both a variable finesse optical resonator and an arbitrary spatial mode converter. By introducing an intracavity electro-optic modulator [18, we anticipate rapid tunability of finesse and output mode, potentially enabling control of photon dynamics within a cavity lifetime.

In our approach, the mode conversion bandwidth is set by the cavity linewidth, and so can be increased by scaling down to micro-resonators. Working with small ROC fiber Fabry-Pérots [44] should enable bandwidths up to $\sim 10 \mathrm{GHz}$, and extending these ideas to nanophotonic platforms would allow further bandwidth gains [27, 45].

The techniques introduced in this work can be employed to inter-convert between field profiles of any physical system in which coupled resonators can be realized whose eigenmodes are the desired input- and output- field profiles. Coupling to a twisted optical resonator 46] whose eigenmodes are Laguerre-Gauss (LG) would enable high-efficiency generation of optical orbital angular 
momentum states for optical communication [47. Similarly, the use of astigmagtic cavities would allow control over both mode indices of the $\mathrm{HG}_{m n}$ output. Indeed, these concepts transcend even light: by coupling together phononic resonators with disparate mode structures, it should be possible to deterministically and efficiently reshape acoustic waves [48, 49].

\section{METHODS}

The tunable finesse cavity and optical mode converter each consist of three low-loss dielectric mirrors supplied by LAYERTEC GmbH. All mirrors have fused silica substrates, rear-side anti-reflection coatings with reflectance $R<0.1 \%$ at the operating wavelength of $780 \mathrm{~nm}$, and front-side reflectances as described in the main text. Outer mirrors have concave surfaces while both middle mirrors are flat. Piezoelectric actuators are employed to vary the relative detuning between the two coupled cavities. A $780 \mathrm{~nm}$ distributed feedback laser (Eagleyard $\mathrm{GmbH}$ ) provides light to test both setups. The beam passed through a $5 \mathrm{~m}$ PM single-mode fiber to clean the spatial mode, yielding $15 \mathrm{~mW}$ of output power.

To measure the tunable finesse cavity, the laser frequency is swept across the cavity resonance by modulating the laser current, and the cavity transmission is measured on a fast photodiode (Thorlabs APD120A). At low finesse the frequency width of the transmission peak reflects the cavity linewidth, with frequency scan calibrated against the transmission of $17 \mathrm{MHz}$ sidebands induced by fast laser current modulation. At high finesse the cavity linewidth becomes smaller than the laser linewidth, and a different protocol must be employed. The laser frequency is rapidly swept across the cavity line, and the linewidth is extracted from the ringdown waveform (see Fig. 1. and ref. [40]).

To measure the absolute mode converter efficiency, light is picked off by two calibrated beam samplers before and after the converter and measured on large-area photodiodes (Thorlabs FDS100). The mode profile is measured on a CCD camera.

\section{ACKNOWLEDGEMENTS}

This work was supported primarily by AFOSR grant FA9550-18-1-0317. This work was also supported by the University of Chicago Materials Research Science and Engineering Center, which is funded by National Science Foundation under award number DMR-1420709. M.S. acknowledges support from the NSF GRFP.

\section{AUTHOR CONTRIBUTIONS}

M.S., A.S., D.S., and J.S. conceived the experiment. M.S. and L.T. performed the experiment. M.S. and J.S. developed the theoretical model. M.S. and J.S. drafted the manuscript. All authors contributed to the manuscript.

\section{AUTHOR INFORMATION}

The authors declare no competing interests.
[1] Vaziri, A., Weihs, G. \& Zeilinger, A. Experimental two-photon, three-dimensional entanglement for quantum communication. Phys. Rev. Lett. 89, 240401 (2002).

[2] Krenn, M. et al. Generation and confirmation of a (100× 100)-dimensional entangled quantum system. Proc. Natl. Acad. Sci. 111, 6243-6247 (2014).

[3] Nagali, E. et al. Quantum information transfer from spin to orbital angular momentum of photons. Phys. Rev. Lett. 103, 013601 (2009).

[4] Wang, J. et al. Terabit free-space data transmission employing orbital angular momentum multiplexing. Nat. Photonics 6, 488 (2012).

[5] Bozinovic, N. et al. Terabit-scale orbital angular momentum mode division multiplexing in fibers. Science 340, 1545-1548 (2013).

[6] Richardson, D. J., Fini, J. M. \& Nelson, L. E. Spacedivision multiplexing in optical fibres. Nat. Photonics $\mathbf{7}$, 354 (2013).

[7] Nicholson, J. W. et al. Scaling the effective area of higherorder-mode erbium-doped fiber amplifiers. Opt. Express 20, 24575-24584 (2012).

[8] Mirhosseini, M. et al. High-dimensional quantum cryptography with twisted light. New J. Phys. 17, 033033
(2015).

[9] Fontaine, N. K. et al. Laguerre-gaussian mode sorter. Nat. Commun. 10, 1-7 (2019).

[10] Sweeney, W. R., Chia, W. H. \& Stone, D. A. Theory of reflectionless scattering modes. Preprint at https://arxiv.org/abs/1909.04017 (2019).

[11] Sabry, Y. M., Saadany, B., Khalil, D. \& Bourouina, T. Silicon micromirrors with three-dimensional curvature enabling lensless efficient coupling of free-space light. Light Sci. Appl. 2, e94 (2013).

[12] Xu, Q., Fattal, D. \& Beausoleil, R. G. Silicon microring resonators with $1.5-\mu \mathrm{m}$ radius. Opt. Express 16, 43094315 (2008).

[13] Dai, D., Wang, J. \& Shi, Y. Silicon mode (de) multiplexer enabling high capacity photonic networks-on-chip with a single-wavelength-carrier light. Opt. Lett. 38, 1422-1424 (2013).

[14] Shen, B., Wang, P., Polson, R. \& Menon, R. Integrated metamaterials for efficient and compact freespace-to-waveguide coupling. Opt. Express 22, 2717527182 (2014).

[15] Chen, C.-F. et al. Creating optical near-field orbital angular momentum in a gold metasurface. Nano Lett. 15, 
$2746-2750(2015)$

[16] Burek, M. J. et al. Fiber-coupled diamond quantum nanophotonic interface. Phys. Rev. Appl. 8, 024026 (2017).

[17] Piggott, A. Y. et al. Inverse design and demonstration of a compact and broadband on-chip wavelength demultiplexer. Nat. Photonics 9, 374-377 (2015).

[18] Huang, Q., Song, G., Chen, J., Shu, Z. \& Yu, J. Proposal and fabrication of an electrooptically controlled multimode microresonator for continuous fast-to-slow light tuning. IEEE Photonics J. 6, 1-11 (2014).

[19] Soltani, M. et al. Efficient quantum microwave-to-optical conversion using electro-optic nanophotonic coupled resonators. Phys. Rev. A 96, 043808 (2017).

[20] Beijersbergen, M. W., Coerwinkel, R. P. C., Kristensen, M. \& Woerdman, J. P. Helical-wavefront laser beams produced with a spiral phaseplate. Opt. Commun. 112, 321-327 (1994).

[21] Demas, J., Rishøj, L. \& Ramachandran, S. Free-space beam shaping for precise control and conversion of modes in optical fiber. Opt. Express 23, 28531-28545 (2015).

[22] Bolduc, E., Bent, N., Santamato, E., Karimi, E. \& Boyd, R. W. Exact solution to simultaneous intensity and phase encryption with a single phase-only hologram. Opt. Lett. 38, 3546-3549 (2013).

[23] Zupancic, P. et al. Ultra-precise holographic beam shaping for microscopic quantum control. Opt. Express 24, 13881-13893 (2016).

[24] Mirhosseini, M. et al. Rapid generation of light beams carrying orbital angular momentum. Opt. Express 21, 30196-30203 (2013).

[25] Granata, M., Buy, C., Ward, R. \& Barsuglia, M. Higherorder laguerre-gauss mode generation and interferometry for gravitational wave detectors. Phys. Rev. Lett. 105, 231102 (2010).

[26] Beijersbergen, M. W., Allen, L., Van der Veen, H. E. L. O. \& Woerdman, J. P. Astigmatic laser mode converters and transfer of orbital angular momentum. Opt. Commun. 96, 123-132 (1993).

[27] Lu, J. \& Vučković, J. Objective-first design of highefficiency, small-footprint couplers between arbitrary nanophotonic waveguide modes. Opt. Express 20, 72217236 (2012).

[28] Dai, D. \& Mao, M. Mode converter based on an inverse taper for multimode silicon nanophotonic integrated circuits. Opt. Express 23, 28376-28388 (2015).

[29] Lai, K., Leon-Saval, S., Witkowska, A., Wadsworth, W. J. \& Birks, T. A. Wavelength-independent all-fiber mode converters. Opt. Lett. 32, 328-330 (2007).

[30] Leon-Saval, S. G. et al. Mode-selective photonic lanterns for space-division multiplexing. Opt. Express 22, 10361044 (2014).

[31] Berkhout, G. C. G., Lavery, M. P. J., Courtial, J., Beijersbergen, M. W. \& Padgett, M. J. Efficient sorting of orbital angular momentum states of light. Phys. Rev. Lett. 105, 153601 (2010).

[32] Labroille, G. et al. Efficient and mode selective spatial mode multiplexer based on multi-plane light conversion. Opt. Express 22, 15599-15607 (2014).

[33] Huang, H. et al. Mode division multiplexing using an orbital angular momentum mode sorter and mimo-dsp over a graded-index few-mode optical fibre. Sci. Rep. 5, 14931 (2015).
[34] Ruffato, G. et al. A compact diffractive sorter for highresolution demultiplexing of orbital angular momentum beams. Sci. Rep. 8, 1-12 (2018).

[35] Miller, D. A. B. Self-configuring universal linear optical component. Photonics Res. 1, 1-15 (2013).

[36] Ribeiro, A., Ruocco, A., Vanacker, L. \& Bogaerts, W. Demonstration of a $4 \times 4$-port universal linear circuit. Optica 3, 1348-1357 (2016).

[37] Ramachandran, S., Wang, Z. \& Yan, M. Bandwidth control of long-period grating-based mode converters in fewmode fibers. Opt. Lett. 27, 698-700 (2002).

[38] Li, S., Mo, Q., Hu, X., Du, C. \& Wang, J. Controllable all-fiber orbital angular momentum mode converter. Opt. Lett. 40, 4376-4379 (2015).

[39] del Campo, A. Shortcuts to adiabaticity by counterdiabatic driving. Phys. Rev. Lett. 111, 100502 (2013).

[40] Poirson, J., Bretenaker, F., Vallet, M. \& Le Floch, A. Analytical and experimental study of ringing effects in a fabry-perot cavity. application to the measurement of high finesses. J. Opt. Soc. Am. B 14, 2811-2817 (1997).

[41] Siegman, A. E. Lasers (University Science Books, Sausalito, 1986).

[42] Hood, C. J., Kimble, H. J. \& Ye, J. Characterization of high-finesse mirrors: Loss, phase shifts, and mode structure in an optical cavity. Phys. Rev. A 64, 033804 (2001).

[43] Hild, S. et al. Measurement of a low-absorption sample of oh-reduced fused silica. Appl. Opt. 45, 7269-7272 (2006).

[44] Hunger, D. et al. A fiber fabry-perot cavity with high finesse. New J. Phys. 12, 065038 (2010).

[45] Lu, J., Boyd, S. \& Vučković, J. Inverse design of a threedimensional nanophotonic resonator. Opt. Express 19, 10563-10570 (2011).

[46] Schine, N., Ryou, A., Gromov, A., Sommer, A. \& Simon, J. Synthetic landau levels for photons. Nature 534, 671675 (2016).

[47] Willner, A. E. et al. Optical communications using orbital angular momentum beams. Adv. Opt. Photonics 7, 66-106 (2015).

[48] Whiteley, S. J. et al. Spin-phonon interactions in silicon carbide addressed by gaussian acoustics. Nature Phys. 15, 490-495 (2019).

[49] Achilleos, V., Theocharis, G., Richoux, O. \& Pagneux, V. Non-hermitian acoustic metamaterials: Role of exceptional points in sound absorption. Phys. Rev. B 95, 144303 (2017).

[50] Mason, S. J. Feedback theory-some properties of signal flow graphs. Proc. IRE 41, 1144-1156 (1953).

[51] Riegle, D. \& Lin, P. Matrix signal flow graphs and an optimum topological method for evaluating their gains. IEEE Trans. Circuit Theory 19, 427-435 (1972).

[52] Arfken, G. B., Weber, H. J. \& Harris, F. E. Mathematical methods for physicists (Academic Press, Waltham, 2011), 7 edn.

[53] Zhao, Z., Guo, C. \& Fan, S. Connection of temporal coupled-mode-theory formalisms for a resonant optical system and its time-reversal conjugate. Phys. Rev. A 99, 033839 (2019).

[54] Suh, W., Wang, Z. \& Fan, S. Temporal coupled-mode theory and the presence of non-orthogonal modes in lossless multimode cavities. IEEE J. Quantum Electron. 40, 1511-1518 (2004).

[55] Gardiner, C. W. \& Collett, M. J. Input and output in damped quantum systems: Quantum stochastic differential equations and the master equation. Phys. Rev. A 31, 
3761 (1985).

[56] Haus, H. A. Waves and fields in optoelectronics (Prentice-Hall, Englewood Cliffs, 1984).

[57] Lang, R. J. \& Yariv, A. An exact formulation of coupledmode theory for coupled-cavity lasers. IEEE J. Quantum Electron. 24, 66-72 (1988). 


\section{Appendix A: Single-Mode S-Matrix Approach for Coupled Fabry Pérot Cavities}

The behavior of a general linear coupled cavity system may be exactly analyzed with a scattering (S) matrix approach, so long as the paraxial and scalar field approximations are valid. In this section only a single spatial mode will be considered. The light field in a given transverse plane may then be described as an amplitude of a right- and a left-traveling wave, or a vector $\boldsymbol{\psi}=\left(\psi_{r}, \psi_{l}\right)^{T}$.

For a region of space containing paraxial optical elements between two transverse planes, there exists a mapping between the incoming waves on either side to the outgoing waves, called the scattering matrix. With the two sides labeled A and B, the scattering matrix $\boldsymbol{S}$ is defined by:

$$
\left[\begin{array}{l}
\boldsymbol{\psi}_{A, \text { out }} \\
\boldsymbol{\psi}_{B, \text { out }}
\end{array}\right]=\left[\begin{array}{ll}
S_{11} & S_{12} \\
S_{21} & S_{22}
\end{array}\right]\left[\begin{array}{l}
\boldsymbol{\psi}_{A, \text { in }} \\
\boldsymbol{\psi}_{B, \text { in }}
\end{array}\right]
$$

The scattering matrices for simple optical elements like on-axis mirrors and regions of free propagation are well known. When multiple optical elements are placed in succession, the overall scattering matrix can be calculated by the transfer matrix approach, or equivalently by pairwise application of the cascaded scattering matrix formula:

$$
\boldsymbol{S}^{\text {tot }}=\left[\begin{array}{cc}
S_{11}^{1}+S_{12}^{1} S_{11}^{2} F S_{21}^{1} & S_{12}^{1}\left(1+S_{11}^{2} F S_{22}^{1}\right) S_{12}^{2} \\
S_{21}^{2} F S_{21}^{1} & S_{22}^{2}+S_{21}^{2} F S_{22}^{1} S_{12}^{2}
\end{array}\right]
$$

with $F=\left(1-S_{22}^{1} S_{11}^{2}\right)^{-1}$.

This formula is sufficient to calculate the overall behavior of a single-mode paraxial system. Using the elementary scattering matrices for free propagation, $\boldsymbol{P}=\left[\begin{array}{cc}0 & e^{i \phi / 2} \\ e^{i \phi / 2} & 0\end{array}\right]$ and mirrors, $\boldsymbol{M}=\left[\begin{array}{cc}r & i t \\ i t & r\end{array}\right]$, we obtain the scattering matrix for a Fabry-Pérot resonator:

$$
\boldsymbol{S}^{F P}=\left[\begin{array}{cc}
r_{1}-\frac{e^{i \phi} t_{1}^{2} r_{2}}{1-e^{i \phi} r_{1} r_{2}} & -\frac{e^{\frac{i \phi}{2}} t_{1} t_{2}}{1-e^{i \phi} r_{1} r_{2}} \\
-\frac{e^{\frac{i \phi}{2}} t_{1} t_{2}}{1-e^{i \phi} r_{1} r_{2}} & r_{2}-\frac{e^{i \phi} t_{2}^{2} r_{1}}{1-e^{i \phi} r_{1} r_{2}}
\end{array}\right]
$$

where $\phi$ is the round-trip phase accrued in the cavity and $r_{i}, t_{i}$ are the field reflection and transmission coefficients. For two coupled Fabry-Pérots the transmitted component is

$$
S_{11}=\frac{-i e^{\frac{1}{2} i\left(\phi_{1}+\phi_{2}\right)} t_{1} t_{2} t_{3}}{1-e^{i \phi_{1}} r_{1} r_{2}-e^{i \phi_{2}} r_{2} r_{3}+e^{i\left(\phi_{1}+\phi_{2}\right)} r_{1} r_{3}\left(r_{2}^{2}+t_{2}^{2}\right)}
$$

An effective finesse for the primary cavity can be extracted by putting Equation A.4 in the usual form of transmission through a Fabry-Pérot, $E_{t} / E_{i}=-\frac{e^{\frac{i \phi_{1}}{2}} t_{1} t_{23}}{1-g e^{i \phi 1}}$, with round-trip gain:

$$
g=r_{1} \frac{r_{2}-r_{3} e^{i \phi_{2}}\left(r_{2}^{2}+t_{2}^{2}\right)}{1-r_{2} r_{3} e^{i \phi_{2}}}
$$

and $t_{23}$ the transmission of the $M_{23}$ cavity. Both of these numbers vary slowly with $\phi_{2}$ in high-finesse configurations. Then the finesse is evaluated as [41] $\mathcal{F}=\frac{\pi \sqrt{|g|}}{1-|g|}$.

The effect of mirror loss on the transmitted field can be easily calculated. Loss in the outer mirrors $M_{1}$ and $M_{3}$ simply reduces the transmitted power by a factor of $\frac{T_{1}}{1-R_{1}} \frac{T_{3}}{1-R_{3}}$, where $1-R_{i}$ is the power transmission of a lossless 
mirror with the same reflectance. To treat loss in $M_{2}$, we note that Equation A.4 is invariant under the substitution:

$$
\begin{aligned}
& r_{2} \rightarrow r_{2}^{\prime}=\frac{r_{2}}{\beta} \\
& t_{2} \rightarrow t_{2}^{\prime}=\frac{t_{2}}{\beta} \\
& r_{1} \rightarrow r_{1}^{\prime}=\beta r_{1} \\
& r_{3} \rightarrow r_{3}^{\prime}=\beta r_{3} \\
& t_{1} \rightarrow t_{1}^{\prime}=\beta t_{1} \\
& \beta^{2}=r_{2}^{2}+t_{2}^{2}=1-L_{2}
\end{aligned}
$$

Thus the transmitted field of a cavity with lossy $M_{2}$ is equivalent to a cavity with lossless $M_{2}$ and modified $M_{1}$ and $M_{3}$. This cavity has spectral properties set by $R_{1}^{\prime}, R_{2}^{\prime}, R_{3}^{\prime}$ and also a loss-induced amplitude reduction given by $\frac{T_{1}^{\prime}}{1-R_{1}^{\prime}} \frac{T_{3}^{\prime}}{1-R_{3}^{\prime}}=\beta \frac{T_{1}}{1-\beta^{2} R_{1}} \frac{T_{3}}{1-\beta^{2} R_{3}} \approx \frac{T_{1}}{T_{1}+L_{1}+L_{2}} \frac{T_{3}}{T_{3}+L_{3}+L_{2}}$ in the high reflectance limit.

Multielement scattering systems may also be treated as a signal flow graph and efficiently solved with Mason's gain formula [50].

\section{Appendix B: Multimode Scattering Matrix Approach for Coupled Fabry-Pérot Cavities}

A general paraxial system may be analyzed by the same scattering matrix approach with simple modifications. For simplicity this discussion will use a single transverse dimension, but the approach is easily extended to a full 2D transverse treatment. The light field in a given transverse plane may be decomposed into a basis of orthonormal Hermite-Gauss mode amplitudes for right- and left-traveling waves, described by a vector $\boldsymbol{\psi}=\left(\psi_{0, r}, \psi_{1, r}, \ldots, \psi_{n, r}, \psi_{0, l}, \psi_{1, l}, \ldots, \psi_{n, l}\right)^{T}=\left(\boldsymbol{\psi}_{r}, \boldsymbol{\psi}_{l}\right)^{T}$. It is accurate to restrict to a finite number $n$ modes so long as the field distribution is bounded and nonsingular.

There is an infinite family of such Hermite-Gauss decompositions parameterized by the "complex beam parameter" $q$ and an axis around which the modes are centered. $q$ determines both the scaling (or waist) of the basis functions, and the degree of wavefront curvature.

Equations A.1 and A.2 are both valid in the multimode case, but with the entries $S_{i j}$ understood to be block matrices of size $n \times n$. As long as the multimode scattering matrices of the individual optical elements are known, the overall scattering matrix may be calculated.

In the Hermite-Gauss basis, the scattering matrices of paraxial optical elements, such as free-space propagation and on-axis mirrors, have a simple form. Such elements do not produce mixing between modes, only overall rescaling and wavefront curvature, amounting to a change in the $q$ parameter [41. Put another way, the block elements $S_{i j}$ of the scattering matrix are diagonal, so long as it is understood that the fields on each port of the interface are expressed in Hermite-Gauss bases with the appropriate $q$ values. We could express the vector with reference to its basis $\psi=\left(\psi_{r, q_{r}}, \psi_{l, q_{l}}\right)^{T}$, but this will be left implicit in our equations. The required relation between $q$ values on each port of a paraxial element can be calculated using the ABCD matrix formalism, but it is not necessary for this discussion.

Inside an optical resonator, there exists a particular choice of $q$ which is transformed back into itself after each round trip [41. This is the most convenient choice, and it guarantees that every element of the resonator is described in a diagonal basis for most resonators, including all two-mirror resonators.

However, the mode converter consists of two optical resonators which have mismatched optical axes and/or waists, so no choice of optical axis and $q$ will yield diagonal forms for the scattering matrices of all elements. One solution is to describe the third mirror as an off-axis mirror whose scattering matrix has mode-mixing terms. Instead, we add an explicit change-of-basis matrix at the interface between the two resonators. This does not represent a physical optical element, but a mathematical transformation which allows the field on either side to be written in different bases. The matrix conveniently casts the field inside each resonator in terms of the eigenmodes of that resonator. The elements of the change-of-basis scattering matrix take the form

$$
\begin{aligned}
S_{12, m n} & =\left\langle\psi_{m} \mid \psi_{n}\right\rangle \\
S_{21} & =S_{12}^{\dagger} \equiv K^{\dagger} \\
S_{11} & =0 \\
S_{22} & =0
\end{aligned}
$$



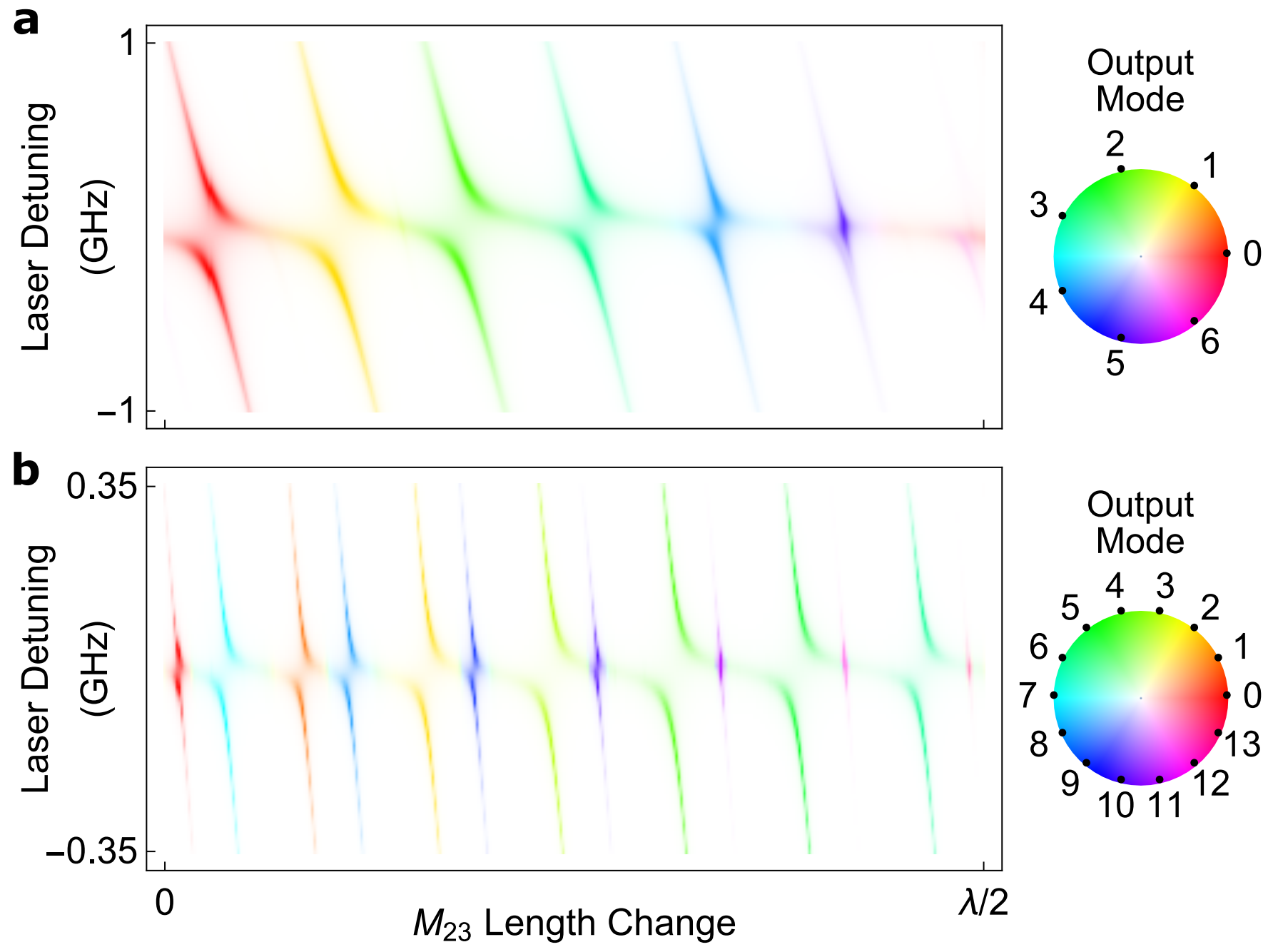

FIG. 4. Simulated Spectrum of Coupled Multimode Optical Resonators. a, Simulated transmission of the mode converter demonstrated in the main text, with $T_{1}=T_{3}=0.035, T_{2}=0.028$, and an input HG $\mathrm{H}_{00}$ beam. The output mode content (indicated by hue) varies as the length of the $M_{23}$ cavity is tuned, bringing different HG $\mathrm{H}_{m 0}$ modes near resonance with the primary cavity. The output power (indicated by color saturation) reaches near unity at the impedance matched condition, as confirmed by the measured cross-sections in Fig. 3. corresponding to perfect mode conversion. Successive mode orders display reduced peak splitting, reflecting a reduced coupling to the $\mathrm{HG}_{00}$ mode of the primary cavity. After $\mathrm{HG}_{50}$ the splitting is smaller than the cavity linewidth and impedance matching cannot be achieved, so higher-order modes disappear from the spectrum. The coupling coefficients are set by the transverse offset between cavities, here 1.3 mode waists. b, Increasing mirror reflectance $\left(T_{1}=T_{2}=T_{3}=0.01\right)$ and transverse offset ( 2.1 mode waists) allows more modes to be impedance matched, and reduces leakage into accidentally near-degenerate modes. An input $\mathrm{HG}_{00}$ can be coherently converted into $\mathrm{HG}_{00}-\mathrm{HG}_{13,0}$. Modes $\mathrm{HG}_{70}-\mathrm{HG}_{13,0}$ belong to the next lower axial mode group and appear interspersed amongst $\mathrm{HG}_{00}-\mathrm{HG}_{60}$.

where the overlap integral $\left\langle\psi_{m} \mid \psi_{n}\right\rangle$ between Hermite-Gauss modes with different optical axis and $q$ may be calculated numerically, or analytically using the method in Appendix D.

The scattering matrices for paraxial elements with the correct $q$ are as follows. Mirrors act as $n$ copies of the form $\boldsymbol{M}$ from Appendix A on the individual modes. Propagation through free space gives a phase shift $P_{12, m n}=S_{21, m n}=$ $\delta_{m n} e^{i[k L+(m+1) \theta]}$, where $\theta$ is the well-known Gouy phase which may be calculated from $q$ and $L$.

The overall scattering matrix for mode-mismatched coupled cavities may be found from repeated application of Equation A.2. The left-to-right transmission is:

$$
\boldsymbol{S}_{21}=-i t_{1} t_{2} t_{3} e^{i \boldsymbol{\Phi}_{\mathbf{2}} / 2} \boldsymbol{K}\left[\boldsymbol{I}-r_{1} r_{2} e^{i \boldsymbol{\Phi}_{\mathbf{1}}}-r_{2} r_{3} \boldsymbol{K}^{\dagger} e^{i \boldsymbol{\Phi}_{\mathbf{2}}} \boldsymbol{K}+\left(r_{2}^{2}+t_{2}^{2}\right) r_{1} r_{3} e^{i \boldsymbol{\Phi}_{1}} \boldsymbol{K}^{\dagger} e^{i \boldsymbol{\Phi}_{\mathbf{2}}} \boldsymbol{K}\right]^{-1} e^{i \boldsymbol{\Phi}_{1} / 2}
$$


and the left-to-left reflection is:

$$
\begin{aligned}
& \boldsymbol{S}_{11}=r_{1} \boldsymbol{I}-t_{1}^{2} e^{i \boldsymbol{\Phi}_{1} / 2}\left[r_{2} \boldsymbol{I}-\left(r_{2}^{2}+t_{2}^{2}\right) r_{3} \boldsymbol{K}^{\dagger} e^{i \boldsymbol{\Phi}_{2}} \boldsymbol{K}\right] \times \\
& \quad\left[\boldsymbol{I}-r_{1} r_{2} e^{i \boldsymbol{\Phi}_{1}}-r_{2} r_{3} \boldsymbol{K}^{\dagger} e^{i \boldsymbol{\Phi}_{2}} \boldsymbol{K}+\left(r_{2}^{2}+t_{2}^{2}\right) r_{1} r_{3} e^{i \boldsymbol{\Phi}_{1}} \boldsymbol{K}^{\dagger} e^{i \boldsymbol{\Phi}_{\mathbf{2}}} \boldsymbol{K}\right]^{-1} e^{i \boldsymbol{\Phi}_{1} / 2}
\end{aligned}
$$

where $\boldsymbol{\Phi}_{i}$ is the round trip propagation matrix for cavity $i$, including Gouy phases.

The mode converter of the main text is simulated using the S-matrix formalism in Fig. 4. Near-unity efficiency mode conversion is predicted when different modes of the two cavities are near resonant, as measured experimentally in Fig. 3 .

Multielement, multimode cavities may also be treated as noncommutative signal-flow graphs with matrix-valued weights and efficiently solved with Riegle's rule [51].

\section{Appendix C: Mode Purity}

Mode purity can be calculated exactly using the S-matrix formalism, or estimated from the mirror transmission coefficients. Mode purity is degraded due to imperfectly suppressed outcoupling through parasitic modes of the $M_{23}$ cavity. The mode in the primary cavity sees outcoupling through the target mode $T_{23}^{i \leftrightarrow j}$, which is set approximately equal to $T_{1}$ in the impedance matched condition. It also sees outcoupling through each unwanted mode $p$ equal to

$T_{23}^{i \leftrightarrow p}=\left|\alpha_{i p}\right|^{2} T_{2} T_{3} f_{p}(\omega)$, where $\alpha_{i p}$ is the mode overlap integral and $f_{p}(\omega)$ is a resonant enhancement factor, which is of order unity if the mode is moderately detuned. The ratio between power in parasitic mode $p$ and the target mode $j$ therefore scales as $\left|\alpha_{i p}\right|^{2} \frac{T_{2} T_{3}}{T_{1}} f_{p}(\omega)$. We note that the sum of all overlap integrals is bounded as $\sum_{k}\left|\alpha_{i k}\right|^{2}=1$. This estimate neglects the second-order effect of additional modes excited in the primary cavity.

\section{Appendix D: Overlap of Hermite-Gauss Modes}

To analyze coupled resonators which are not spatially mode-matched, it is useful to perform a change of basis between their eigenmodes. This transformation requires the overlap integrals between offset and/or rescaled HermiteGauss (HG) functions. These integrals may be calculated numerically, in which case it is useful to calculate the Hermite polynomials using a stable algorithm such as the recurrence relation [52 $H_{n+1}(x)=2 x H_{n}(x)-2 n H_{n-1}(x)$. They can also be calculated analytically using the method of generating functions. Here we work in a single transverse dimension for simplicity. The normalized Hermite-Gauss functions $\mathrm{HG}_{n}$ with waist $w$ are given by:

$$
\mathrm{HG}_{n}(x ; w)=\sqrt{\frac{\sqrt{2 / \pi}}{2^{n} n ! w}} H_{n}\left(\frac{\sqrt{2} x}{w}\right) e^{-\frac{x^{2}}{w^{2}}}
$$

where $H_{n}(x)$ is the $n$ th-order Hermite polynomial and the HG functions are taken to have no wavefront curvature (valid when the overlap is taken at the mode waist). The generating function for unnormalized HG functions is:

$$
\begin{aligned}
g_{w}(x, t) & =e^{\frac{2 \sqrt{2} x}{w} t-t^{2}} e^{-\frac{x^{2}}{w^{2}}} \\
& =\sum_{n=0}^{\infty} H_{t}\left(\frac{\sqrt{2} x}{w}\right) e^{-\frac{x^{2}}{w^{2}}} \frac{t^{n}}{n !}
\end{aligned}
$$

The overlap integral between modes $m$ and $n$ of two HG bases is calculated by taking the integral of the product of their respective generating functions, picking off the correct series coefficients, and inserting normalization factors:

$$
\begin{aligned}
\int \mathrm{HG}_{m, \lambda w}^{*}(x) \mathrm{HG}_{n, w}(x+a w) d x= & \sqrt{\frac{2}{\pi 2^{m} m ! 2^{n} n ! \lambda w^{2}}}\left[\frac{d^{m}}{d u^{m}} \frac{d^{n}}{d t^{n}} \int g_{\lambda w}^{*}(x, u) g_{w}(x+a, t) d x\right]_{u, t=0} \\
= & \sqrt{\frac{2}{2^{m} m ! 2^{n} n !}} \sqrt{\frac{\lambda}{1+\lambda^{2}} e^{-\frac{a^{2}}{1+\lambda^{2}}} \times} \\
& \left\{\frac{d^{m}}{d u^{m}} \frac{d^{n}}{d t^{n}} \operatorname{Exp}\left[\frac{\left(1-\lambda^{2}\right)\left(t^{2}-u^{2}\right)+4 t u \lambda+2 \sqrt{2} a(u-\lambda t)}{1+\lambda^{2}}\right]\right\}_{u, t=0}
\end{aligned}
$$


In this work we only require the overlap of modes with zero wavefront curvature. However, a similar derivation applies more generally, using the complex $q$-parameter formulation of the Hermite-Gauss functions.

\section{Appendix E: Two-Mode Coupled Mode Analysis}

In Appendix $\mathrm{F}$ we derive coupling constants for the phenomenological temporal coupled mode theory (TCMT) treatment of multimode coupled optical cavities. Here we use the results for a simple case with two transversely offset optical resonators coupled through a shared mirror, each supporting a single mode (which may have different transverse profiles). Assuming reciprocal media and neglecting loss, the scattering matrix takes the form [53, 54]:

$$
S=-I-i \boldsymbol{D} \frac{1}{(\boldsymbol{\Omega}-i \boldsymbol{\Gamma})-\omega} \boldsymbol{D}^{T}
$$

with

$$
\begin{aligned}
& \boldsymbol{\Omega}=\left[\begin{array}{cc}
-\frac{\delta}{2} & g \\
g & \frac{\delta}{2}
\end{array}\right] \\
& \boldsymbol{D}=\left[\begin{array}{cc}
\sqrt{\gamma_{1}} & 0 \\
0 & \sqrt{\gamma_{2}}
\end{array}\right] \\
& \boldsymbol{\Gamma}=\boldsymbol{D}^{\dagger} \boldsymbol{D}=\left[\begin{array}{cc}
\frac{\gamma_{1}}{2} & 0 \\
0 & \frac{\gamma_{2}}{2}
\end{array}\right]
\end{aligned}
$$

with $\delta$ the detuning between the modes. The coupling rate between the resonator mode in cavity $i$ and its corresponding output channel is $\sqrt{\gamma_{i}}=-\log R_{i} \nu_{i}$, where $\nu_{i}$ is the free spectral range and $R_{i}$ is the reflectance of the output mirror. The coupling rate between the two resonator modes is $g=\alpha \sqrt{-\log R_{c} \nu_{1} \nu_{2}}$, where $\alpha$ is the overlap integral between the two modes and $R_{c}$ is the reflectance of the shared mirror.

Impedance matching occurs when the reflection coefficient vanishes. In the energy-conserving case, transmission reaches unity at this point, indicating full mode conversion. With matched cavity decay rates $\gamma_{1}=\gamma_{2}=\gamma$, this occurs at $\delta=0, \omega= \pm \sqrt{g^{2}-\left(\frac{\gamma}{2}\right)^{2}}$. Under these conditions, evaluation of the eigenmodes of the effective Hamiltonian, $\boldsymbol{\Omega}-i \boldsymbol{\Gamma}$, shows that equal stored energy resides in each cavity.

For $g<\frac{\gamma}{2}$ there is no real solution, but the minimum reflection occurs at $\delta=0, \omega=0$. Thus a solution with unit efficiency mode conversion exists whenever $|\alpha|^{2}>\frac{\gamma^{2}}{-4 \log R_{c} \nu_{1} \nu_{2}}$.

For mismatched cavity decay rates $\gamma_{1} \neq \gamma_{2}$, impedance matching occurs at $\delta \neq 0$, but there is still generally a solution for sufficiently large $|\alpha|$. In this case, the product of the stored energy and the decay constant of each cavity is equal.

\section{Appendix F: Multimode Coupled Mode Analysis}

The S-matrix analysis of Appendix B relies only on the paraxial and scalar field approximations and is otherwise exact. Coupled optical cavities can also be analyzed using the temporal coupled mode theory (TCMT), a phenomenological model of open resonant optical systems. Although TCMT is not derived from first principles, it has been shown to agree well with rigorous analysis and provides useful intuition for the design of optical devices. TCMT is mathematically equivalent to the input-output formalism of damped quantum systems [55]. Here a full multimode theory for coupled optical resonators will be developed, while Appendix E specializes to the two-mode limit to discuss impedance matching.

In this formalism an optical cavity is described by a set of $M$ cavity modes which are allowed to couple with each other and with $N$ ports, each containing an incoming and outgoing propagating channel. Assuming reciprocal media, the coupled mode equations are [10, 53, 54] :

$$
\begin{aligned}
\frac{d}{d t} \boldsymbol{a} & =-i(\boldsymbol{\Omega}-i \boldsymbol{\Gamma}) \boldsymbol{a}+\boldsymbol{D}^{T} \boldsymbol{s}_{+} \\
\boldsymbol{s}_{-} & =\boldsymbol{C} \boldsymbol{s}_{+}+\boldsymbol{D} \boldsymbol{a}
\end{aligned}
$$


where $\boldsymbol{a}$ is a state vector containing the $M$ amplitudes of the modes, normalized such that $\left|a_{i}\right|^{2}$ corresponds to the energy stored in the $i$ th mode. $\boldsymbol{\Omega}$ and $\boldsymbol{\Gamma}$ are $M \times M$ Hermitian matrices, with $\boldsymbol{\Omega}$ representing the resonator mode frequencies and couplings and $\boldsymbol{\Gamma}$ representing decay processes. The resonances are coupled to the $N$ incoming channels $\boldsymbol{s}_{+}$and outgoing channels $\boldsymbol{s}_{-}$according to the coefficients in the $N \times M$ matrix $\boldsymbol{D}$. The channel amplitudes are normalized such that $\left|s_{+i}\right|^{2}\left(\left|s_{-i}\right|^{2}\right)$ is the power carried by the $i$ th incoming (outgoing) channel. The $N \times N$ symmetric matrix $\boldsymbol{C}=\boldsymbol{C}^{T}$ represents direct coupling from input to output channels, including direct reflection and processes not included in the resonant modes $\boldsymbol{a}$.

Assuming harmonic time dependence for $\boldsymbol{a}$ then eliminating $\boldsymbol{a}$ from Equations F.1, F.2 gives the S-matrix $\boldsymbol{s}_{-}=\boldsymbol{S} \boldsymbol{s}_{+}$ as:

$$
\boldsymbol{S}=\boldsymbol{C}-i \boldsymbol{D} \frac{1}{(\boldsymbol{\Omega}-i \boldsymbol{\Gamma})-\omega} \boldsymbol{D}^{T}
$$

For systems with no absorption loss, all decay comes from radiative coupling to propagating channels. When energy conservation and time-reversal symmetry hold, it can be shown that [53]:

$$
\begin{aligned}
\boldsymbol{\Gamma} & =\frac{\boldsymbol{D}^{\dagger} \boldsymbol{D}}{2} \\
\boldsymbol{C} \boldsymbol{D}^{*} & =-\boldsymbol{D} \\
\boldsymbol{C}^{\dagger} \boldsymbol{C} & =I
\end{aligned}
$$

In what follows, we neglect loss so that these relationships hold.

All that remains is evaluation of the (system-dependent) coupling constants in $\boldsymbol{C}, \boldsymbol{D}$, and $\boldsymbol{\Omega}$. For two coupled optical resonators, the modes are enumerated as follows. All modes are labeled by their tranverse spatial mode index $t$. Channel modes have an additional port index yielding $s_{ \pm, p t}$. Resonator modes have a cavity index and an axial mode index $z$ yielding $a_{c t z}$.

To define the direct coupling matrix $\boldsymbol{C}$ we note that any incoming power not coupled into the resonator is reflected into the same channel, so $\boldsymbol{C}$ is diagonal. Combined with Equation F.6 this means each element of $\boldsymbol{C}$ is a phase factor with unit magnitude. We take coupling to occur at the mirror surface, so that all transverse modes must experience the same reflection phase shift. This defines $\boldsymbol{C}$ up to a single arbitrary phase, which we choose so that $\boldsymbol{C}=-I$.

The resonator-to-channel matrix $\boldsymbol{D}$ only couples modes with the same spatial mode index $t$. Resonator modes in a given cavity only couple to mirror(s) connected to that cavity. Therefore the element $D_{p t^{\prime}, \text { ctz }}=\xi_{p t z} \delta_{t, t^{\prime}} \sigma_{p, c}$, where we define $\sigma_{p, c}=1$ if port $p$ is connected to cavity $c$ and zero otherwise, and $\xi_{p t z}$ is a complex constant. The magnitude of $\xi_{p t z}$ is fixed by an energy conservation argument [56. We note that the energy of a single populated mode $a_{c t z}$ with no input decays as $\left|a_{c t z}(t)\right|^{2}=\left|a_{c t z}(0)\right|^{2} e^{-\sum_{p} \gamma_{p} t}$, where the sum is over ports accessible from cavity $c, \gamma_{p}=-\nu_{c} \ln R_{p}$ is the decay rate into port $p, \nu_{c}$ is the free spectral range of cavity $c$, and $R_{p}$ is the reflectance of the mirror at port $p$. The power exiting is $\frac{d}{d t}\left|a_{c t z}(t)\right|^{2}=-\left(\sum_{p} \gamma_{p}\right)\left|a_{c t z}(t)\right|^{2}$. Therefore we ascribe a decay coefficient $\gamma_{p}$ to each port $p$ coupled to $a_{c t z}$. However, according to Equation F.2 the power exiting into port $p$ is $\left|s_{-p t}\right|^{2}=\left|D_{p t, c t z}\right|^{2}\left|a_{c t z}(t)\right|^{2}$. Thus $\left|D_{p t, c t z}\right|^{2}=\gamma_{p}$ and $\left|\xi_{p t z}\right|=\sqrt{\gamma_{p}}$.

The phase of $\xi_{p t z}$ is constrained by Equation F.5 and our choice of $\boldsymbol{C}=-I$, yielding $\boldsymbol{D}^{*}=\boldsymbol{D}$, so all elements of $\boldsymbol{D}$ are real and defined up to a sign. Each resonant mode can have one arbitary sign in the coupling constant at one port. For all other ports accessible to that mode, the sign must be chosen consistently. This is important when multiple axial modes are included; adjacent axial modes have opposite parity, and incorrectly chosen signs will affect the interference between modes.

Finally we evaluate the closed-cavity Hamiltonian matrix $\boldsymbol{\Omega}$. The diagonal elements are just the (real) mode frequencies set by the free spectral range and transverse mode spacings. The off-diagonal elements represent coupling rates between resonant modes. We evaluate these with a similar energy conservation argument as used for $\boldsymbol{D}$ [56]. Coupling occurs between the modes of two cavities separated by a mirror of reflectance $R_{c}$. The circulating power in mode $a_{c^{\prime} t^{\prime} z^{\prime}}$ of cavity $c^{\prime}$ excites a mode $a_{c t z}$ of cavity $c$. According to Equation F.1 the coupling contributes to $\frac{d}{d t} a_{c t z}$ a term $\Omega_{c t z, c^{\prime} t^{\prime} z^{\prime}} a_{c^{\prime} t^{\prime} z^{\prime}}$. This can be compared to excitation of a mode by a propagating channel, which contributes to $\frac{d}{d t} a_{c t z}$ a term $D_{p t, c t z} s_{+p t}$, where the incident power is $P=\left|s_{+p t}\right|^{2}$ and we have already determined the magnitude $\left|D_{p t, c t z}\right|=\sqrt{-\nu_{c} \ln R}$. In the present case the incident power due to mode $a_{c^{\prime} t^{\prime} z^{\prime}}$ is $P=\left|a_{c^{\prime} t^{\prime} z^{\prime}}\right|^{2} \nu_{c^{\prime}}\left|\alpha_{t^{\prime}, t}\right|^{2}$, where the overlap integral $\alpha_{t^{\prime}, t}$ restricts to that portion of the incident mode which is spatially mode-matched. Comparing these two cases, we must have $\left|\Omega_{c t z, c^{\prime} t^{\prime} z^{\prime}}\right|=\sqrt{-\nu_{c} \nu_{c^{\prime}} \ln R_{c}}\left|\alpha_{t^{\prime}, t}\right|$. The phase of the coupling coefficients must be chosen with similar concern as the elements of $\boldsymbol{D}$, taking into account the opposite parity of adjacent axial modes.

Although there exists an exact coupled-mode description of single-mode resonators [57] which could be extended 
to the multimode case, we do not pursue that here, as the S-matrix description of Appendix B provides exact results, and the simpler coupled-mode theory is quite accurate and useful for intuition. 